\title{
Cytokeratins negatively regulate the invasive potential of lung cancer cell lines
}

\author{
NOBUHIRO KANAJI ${ }^{1}$, SHUJI BANDOH ${ }^{1}$, TOMOYA ISHII ${ }^{1}$, \\ JIRO FUJITA $^{2}$, TOSHIHIKO ISHIDA ${ }^{1}$, TAKUYA MATSUNAGA ${ }^{1}$ and AKIHITO KUBO ${ }^{3}$ \\ ${ }^{1}$ Division of Endocrinology and Metabolism, Hematology, Rheumatology and Respiratory Medicine, \\ Department of Internal Medicine, Kagawa University, Kagawa; ${ }^{2}$ Department of Infectious, Respiratory, \\ and Digestive Medicine, Control and Prevention of Infectious Diseases, Faculty of Medicine, \\ University of the Ryukyus, Okinawa; ${ }^{3}$ Division of Respiratory Medicine and Allergology, \\ Department of Internal Medicine, Aichi Medical University School of Medicine, Aichi, Japan
}

Received May 6, 2011; Accepted May 23, 2011

DOI: 10.3892/or.2011.1357

\begin{abstract}
Lung cancer cells express several cytokeratins (CKs) that are subdivided into type I (CK9-23) and type II (CK1-8) subclasses. The functions of CKs in lung cancer cells have not been fully elucidated. The purpose of this study was to investigate the role of CKs in the invasion of lung cancer cells. We investigated the expression levels of CK7, 8, 18 and 19 in 12 non-small cell lung cancer (NSCLC) and seven SCLC cell lines by quantitative immunoblotting. The expression levels of these four CKs were significantly higher in the NSCLC cells. The NSCLC cell line HI1017 expressed CK8 and 18; A549 cells expressed CK7, 8, 18 and 19, respectively. Invasive sublines of HI1017 and A549 were established by repeated selection of invasive cells using a membrane invasion chamber system. The invasive cell lines showed lower expression levels of CKs compared with the parental cells. Exogenous CK19 also resulted in a decrease in invasiveness of the HI1017 cells. Suppression of either CK8 or CK18 by short interfering RNAs led to a decrease in the total CKs and increased invasiveness of both the HI1017 and A549 cells. A549 cells expressed very low levels of CK19. Suppression of CK19 affected neither invasive ability nor total CK amount in the A549 cells. Our observations indicate that $\mathrm{CK}$ expression levels were inversely associated with invasiveness of the NSCLC cell lines, and suggest that expression levels of dominant CKs may affect invasive ability.
\end{abstract}

Correspondence to: Dr Nobuhiro Kanaji, Division of Endocrinology and Metabolism, Hematology, Rheumatology and Respiratory Medicine, Department of Internal Medicine, Faculty of Medicine, Kagawa University, 1750-1 Ikenobe, Miki-cho, Kita-gun, Kagawa 761-0793, Japan

E-mail: kanaji@med.kagawa-u.ac.jp

Key words: cytokeratin, lung cancer, invasion, Matrigel

\section{Introduction}

Cytokeratins (CKs) constitute the largest family of intermediate filaments and are subdivided into type I (CK9-23) and type II (CK1-8) subclasses (1). Various CK combinations are expressed in an epithelial cell-type preferential manner (1). Epithelial cells in the respiratory tract express CK8 and CK18 as dominant CKs with additional type II CKs (CK5, 6, and 7) as well as type I CKs (CK17 and 19) to various extents $(1,2)$.

CKs form non-covalent heteropolymers at a 1:1 molar ratio (type I:type II) and comprise the cytoskeletal network. Neither type I nor type II CKs alone can form proper CK filaments and undergo proteolytic degradation, indicating that both type I and type II CKs are required for proper filament formation $(1,3)$. We recently reported the compensation of type I and II CKs in non-small cell lung cancer (NSCLC) cell lines. Suppression of a single dominant CK causes down-regulation of CKs of the complementary type while suppression of non-dominant CKs does not affect expression of other CKs. These fundamental observations suggest that balanced expression of type I and II CKs is critical for the organization of CK networks (4).

Several functions of CKs have been clarified, including their roles in scaffolding and tissue integrity, by gene knockout studies (1). In addition, CKs have been reported to play an important role in the regulation of cell invasion and tumorigenesis (5-11). In CK19-deficient oral squamous cell carcinoma lines, stable expression of CK19 significantly decreased invasive ability (5). Down-regulated expression of CK18 was significantly correlated with the progression of human breast cancer (6). In contrast, some studies have shown that cells co-expressing CK8, 18 and vimentin have high invasive potential in fibroblasts, melanoma cells, and lung cancer cells (9-11).

Epithelial-mesenchymal transition (EMT) is a process involved in embryonic developmental and also occurs during the progression of several types of cancer and confers invasiveness to cancer cells (12). It has been reported that epithelial markers such as CKs decrease in EMT-induced lung cancer cells (13). 

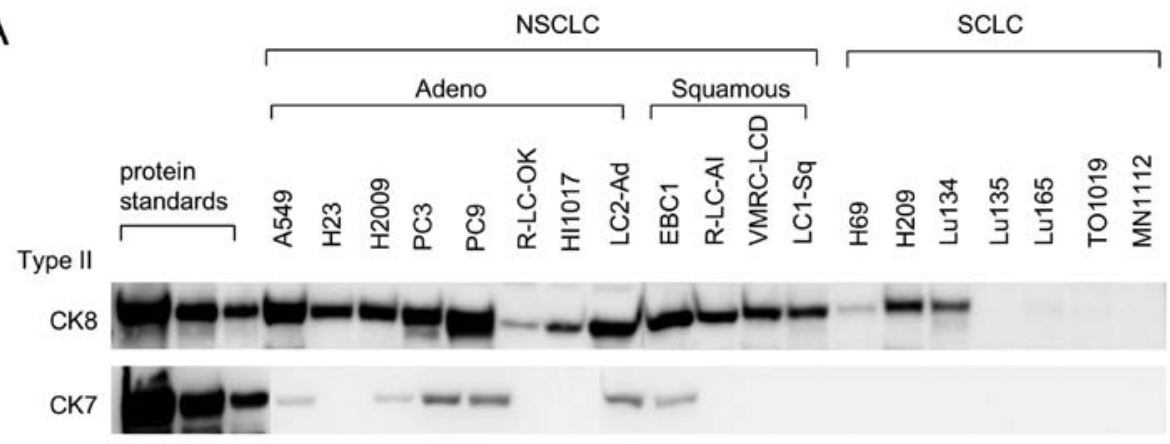

Type I

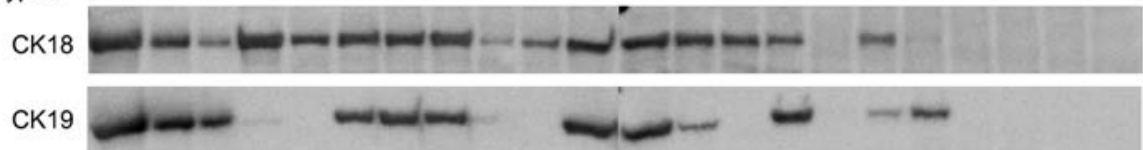

Coomassie staining

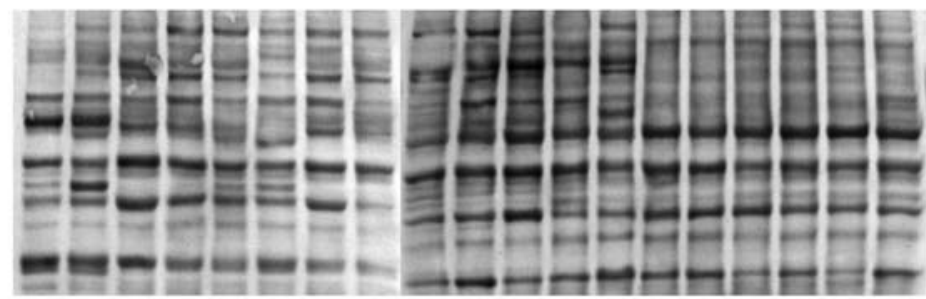

B
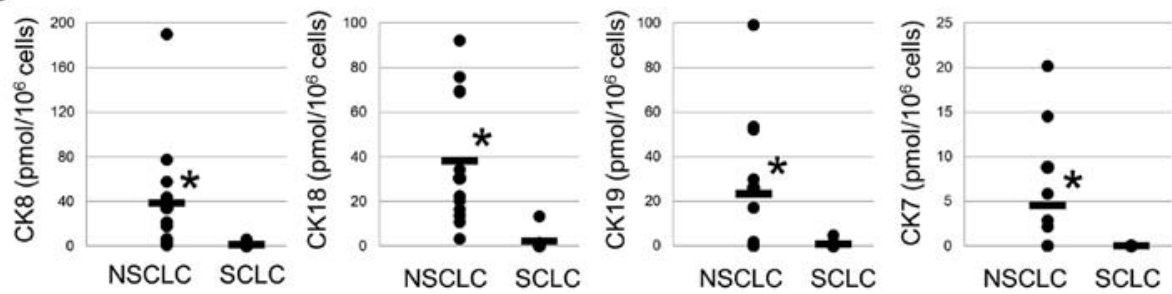

Figure 1. Expression of CKs in non-small cell lung cancer (NSCLC) and small cell lung cancer (SCLC) cell lines. (A) Quantitative immunoblotting for CKs in 12 NSCLC and seven SCLC cell lines. Coomassie Brilliant Blue dye was used for the staining of all proteins. Immunoblots presented are representative of three independent experiments. (B) Quantification of CKs. Absolute expression levels of CKs were quantified by repeated immunoblotting. Dots indicate each cell line and bars show the average values. ${ }^{*} \mathrm{p}<0.05$ compared with SCLC.

Based on these findings, we hypothesized that CKs may play a role in the invasiveness of lung cancer. To investigate this, we established highly invasive lung cancer cells by repeated selection of invasive cells through a membrane coated with a basement membrane preparation (Matrigel ${ }^{\mathrm{TM}}$ ), and assessed the expression of CKs. We also tested the invasive ability in the cells transfected with CKs or in those where $\mathrm{CK}$ was silenced.

\section{Materials and methods}

Cells and reagents. Nineteen lung cancer cell lines (12 NSCLC and 7 SCLC) were obtained from the Japan Cancer Research Bank (Tokyo, Japan). Cells were cultured in RPMI-1640 supplemented with $10 \%$ fetal bovine serum (FBS). The antibodies used were K8.8 (CK8; Neomarkers, Fremont, CA, USA), Ks18.04 (CK18; Progen, Heidelberg, Germany), RCK105 (CK7; Progen), Ks19.02 (CK19; Progen), AC-15 ( $\beta$-actin, Sigma-Aldrich, St. Louis, MO, USA), C5 (GAPDH; Chemicon, Temecula, CA, USA) and anti-mouse immunoglobulin $\mathrm{G}$ (IgG) conjugated with horseradish peroxidase (HRP; Santa Cruz Biotechnology, Santa Cruz, CA, USA). Purified proteins were purchased from Progen (CK8, 18, 19, 20). Full length cDNA of CK7 was cloned into pGEX-2TK
(Pharmacia), and recombinant CK7 was expressed in DH5 $\alpha$ Escherichia coli (Invitrogen, Bethesda, MD, USA). Short interfering RNAs (siRNAs) targeting CKs were purchased from Santa Cruz Biotechnology (sc-35156 for CK8, sc-35151 for CK18, and sc-35152 for CK19).

Quantitative immunoblotting. Exponentially growing cells were counted in triplicate using a hemocytometer, lysed in 1X sample buffer (62.5 mM Tris-HCl pH 6.8, 2.15\% SDS, 5\% $\beta$-mercaptoethanol, $15 \%$ glycerol), and subjected to sodium dodecyl sulfate-polyacrylamide agarose gel electrophoresis (SDS-PAGE) followed by immunoblot analyses using specific antibodies and enhanced chemiluminescence. The intensity of positive signals in the immunoblot analyses was quantified densitometrically using NIH Image version 1.62 (http://rsb. info.nih.gov/nih-image/download.htm). The quantified signal intensities were then compared with those of the protein standards at known concentrations. The PVDF membranes used for immunoblots were stained with Coomassie blue dye.

In vitro invasion assay. The membrane invasion culture system (MICS) was used to assess cell invasion (11). Briefly, $4 \times 10^{5}$ cells suspended in RPMI-1640 without FBS were seeded 
A

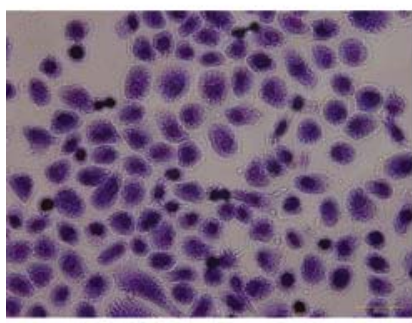

Parent HI1017 (HI1017-0)

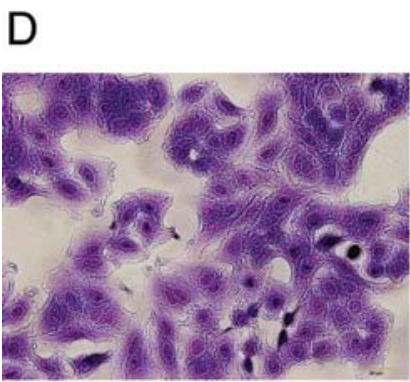

Parent A549 (A549-0)
B

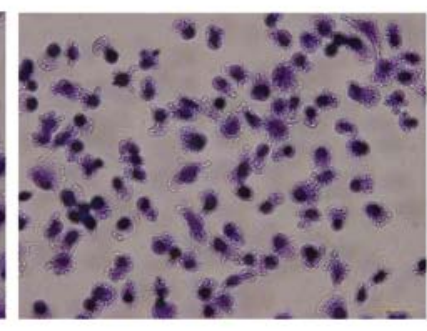

Invasive HI1017 (HI1017-18)

E

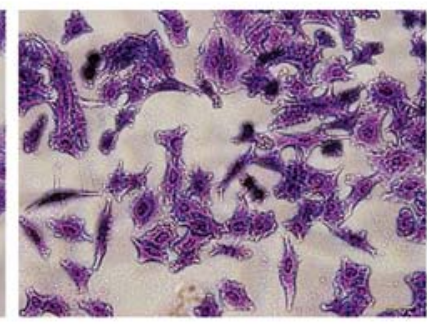

Invasive A549 (A549-18)
C

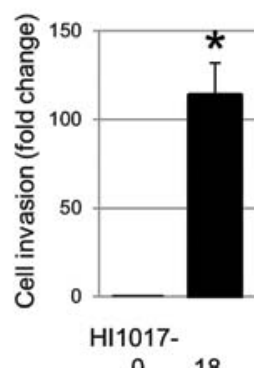

$0 \quad 18$

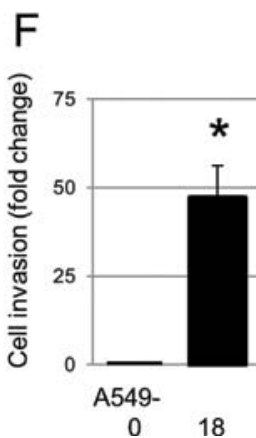

Figure 2. Cell morphology and invasive ability of cells that migrated through the Matrigel. Invasive cells were established by selection of the invasive subpopulation using the membrane invasive culture system (MICS) 18 times. (A) Cell morphology of parent HI1017 (HI1017-0) cells. (B) Changes in cell morphology of invasive HI1017 (HI1017-18) cells. (C) Invasive ability was assessed by in vitro invasion assays using Matrigel-coated membranes. Data presented are the means of fold change \pm SD from three independent experiments. * $\mathrm{p}<0.01$ compared with HI1017-0. (D) Cell morphology of parent A549 (A549-0) cells. (E) Cell morphology of invasive A549 (A549-18) cells. (F) In vitro invasion assay. "p<0.01 compared with A549-0.

into the upper wells of Matrigel-coated invasion chambers (Becton-Dickinson, Franklin Lakes, NJ, USA). RPMI-1640 supplemented with $10 \%$ FBS was added in the lower wells as a chemoattractant. After incubation for $12 \mathrm{~h}$, cells that invaded through the membrane were fixed in methanol, stained and microscopically counted.

Establishment of invasive cells. Subpopulations from cancer cell lines (HI1017 and A549) were selected according to their differential invasiveness using the MICS as previously described, with a slight modification (11). Similar to invasion assays, cells were seeded into the upper wells. Following incubation for $12-72 \mathrm{~h}$ at $37^{\circ} \mathrm{C}$, the inserts were removed. The cells that migrated through the Matrigel membranes and attached to the lower wells were allowed to proliferate. This selection of invasive cells was repeated up to 18 times to establish highly invasive sublines. Parental cells were designated as HI1017-0 or A549-0, and invasive cells that migrated through the Matrigel 18 times were designated HI1017-18 or A549-18.

RNA interference (RNAi). To selectively silence each CK, RNAi was performed. Cells were seeded in 6-well plates at a density of $4 \times 10^{5}$ cells/well. The next day, cells were transfected with siRNA (final concentration $20 \mathrm{nM}$ ) in OptiMEM I (Invitrogen) using Lipofectamine 2000 (Invitrogen). After $8 \mathrm{~h}$ of incubation, the media were replaced with RPMI-1640 supplemented with $10 \%$ FBS. Cell lysates were extracted on day 4 , and the efficacy of RNAi was assessed by immunoblots.

Stable transfection. Cells were transfected with pcDNA-CK19, and selected in the presence of Geneticin (Invitrogen) for stable expression of exogenous CK19, as described previously (4).
Statistical analysis. Data were expressed as the mean \pm standard deviation (SD). An unpaired two-tailed Student's t-test was used for single comparisons. p-values $<0.05$ were considered statistically significant.

\section{Results}

Differential expression of CKS in NSCLC and SCLC cell lines. Expression of CK7, 8, 18 and 19 was first examined in 12 NSCLC and seven SCLC cell lines (Fig. 1A). Other CKs (CK4, 5, 6, 13, 17 and 20) were not detected in these cell lines (data not shown). Absolute expression levels of CKs were quantified by quantitative immunoblotting (Fig. 1B). All NSCLC cell lines tested in the present study expressed type II CK8 and type I CK18 as dominant CKs, 40 pmol per $10^{6}$ cells on average. Of the 12 NSCLC cell lines, eight expressed CK19 and six expressed CK7 at lower levels. Of note, expression levels of each $\mathrm{CK}$ were significantly higher in the NSCLCs compared with the SCLCs $(\mathrm{p}<0.05)$.

Cell morphology and invasive ability of cells that migrated through Matrigel. Invasive subpopulations of lung cancer cell lines were established by the repeated selection of invasive cells using the MICS. When HI1017 cells migrated through Matrigel 18 times (HI1017-18), changes in cell morphology were observed. Typically, HI1017-18 cells were smaller in size, and the cytoplasmic area of these cells was decreased compared with the parental HI1017 cells (HI1017-0; Fig. 2A and B). For the in vitro invasion assay, HI1017-18 cells showed higher invasive ability compared with the parental HI1017 cells (Fig. 2C, 113.7 \pm 18.3 -fold, p<0.01). Similar to HI1017, A549-18 cells were smaller with decreased cytoplasmic area 
A

B
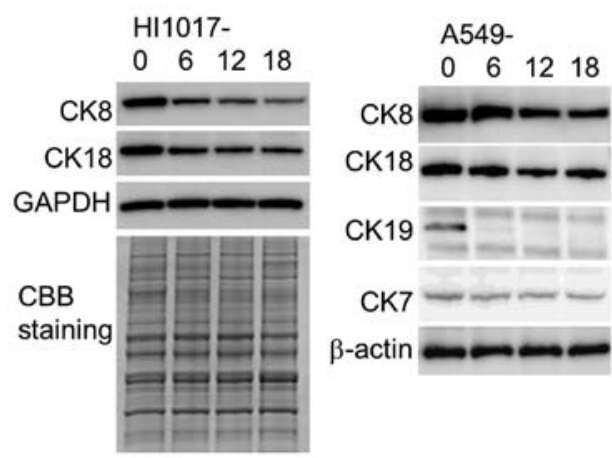

Figure 3. Invasive cells express lower levels of CKs. (A) Expression of CKs in the HI1017 cell line. Cellular proteins were extracted. Immunoblots presented are representative of three independent experiments. Immunoblots for GAPDH and Coomassie brilliant blue (CBB) staining were used as loading controls. (B) Immunoblots for CKs in A549 cells. Repetitive MICS selection decreased CK expression levels without affecting the expression of either $\beta$-actin or GAPDH.

(Fig. 2D and E) and showed greater invasive ability compared with the A549-0 cells (Fig. 2F, p<0.01).

Invasive cells express CKs at lower levels. HI1017 cells express CK8 and CK18 as dominant CKs (Fig. 1). When expression of CKs was assessed in the invasive HI1017 cells (HI1017-6, -12 and -18), a decrease in both CK8 and CK18 was observed (Fig. 3A). A549 cells also showed lower expression levels of CKs (CK7, 8, 18 and 19) when the cells acquired an invasive phenotype (Fig. 3B).

Introduction of exogenous $C K s$ results in a decrease in invasive ability. HI1017 cells lack endogenous CK19. To confirm the effect of CKs on invasive ability, we transfected HI1017 cells with exogenous CK19 (Fig. 4A). CK19-expressing clones 1 and 2 showed lower invasive ability than mocktransfected cells (Fig. 4B).

Suppression of CKs leads to an invasive phenotype. To further investigate the function of CKs on invasiveness, we performed RNAi to silence each CK. Since the only
A
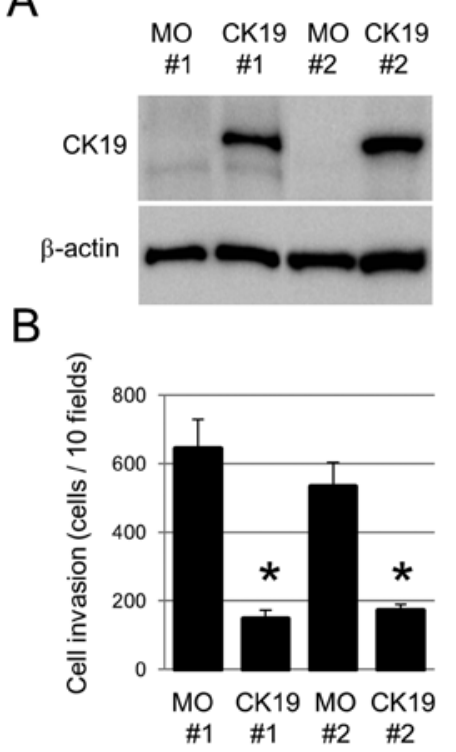

Figure 4. Exogenous CK19 inhibits invasive phenotype. (A) Stable HI1017 clones expressing exogenous CK19 (CK19). (B) Invasive assay. Data presented are the means \pm SD of three independent experiments. " $\mathrm{p}<0.01$ compared with mock (MO) transfections.

CKs expressed in HI1017 were CK8 and CK18, suppression of one CK directly decreased the level of the other CK (4). Therefore, transfection with siRNAs targeting either CK8 or CK18 resulted in suppression of both CKs (Fig. 5A). HI1017 cells lacking CKs showed an $\sim 4$-fold higher invasive phenotype compared with the cells transfected with control-siRNA (Fig. 5B, p<0.05). In the A549 cells, transfection with siRNAs targeting CK18, the dominant type I CK, led to suppression of CK8, 7 and 19 in addition to CK18 (Fig. 5C). A549 cells transfected with this CK18 siRNA showed a $>3$-fold higher invasive ability (Fig. 5D). Transfection with siRNAs targeting CK8, the dominant type II CK, led to suppression of CK8 and its partner type I CKs, CK18 and 19 (Fig. 5C). In addition, the invasive ability of A549 cells transfected with CK8 siRNA was higher (Fig. 5D; 2.8 \pm 0.7 -fold, $\mathrm{p}<0.05$ ). A549 cells express CK19 at trace levels. Transfection with siRNA targeting this minor CK19 did not affect invasive ability.
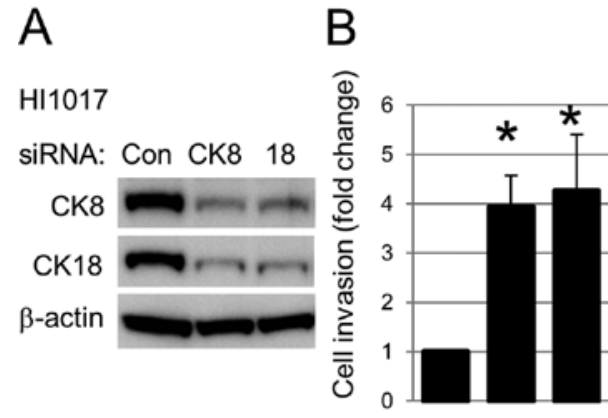

siRNA: Con CK8 CK18
C

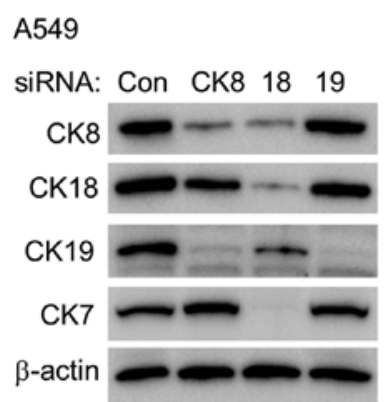

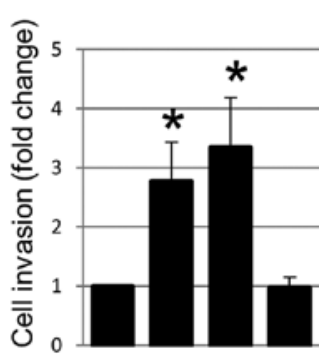

SiRNA: Con CK8 1819

Figure 5. Silencing of CKs leads to an invasive phenotype. HI1017 (A and B) and A549 (C and D) cells were transfected with siRNA targeting CK8, 18 or 19. (A and C) The efficacy of RNA interference was evaluated by immunoblots. (B and D) Invasion assay. Data presented are the means \pm SD of three independent experiments. ${ }^{*}$ p $<0.05$ compared with control siRNA. 


\section{Discussion}

This study demonstrated that i) highly invasive cells expressed lower levels of CKs, ii) suppression of CKs increased cell invasion, and iii) transfection of exogenous CKs resulted in a decrease in invasive phenotype. These data suggest that CKs negatively regulate the invasive potential of lung cancer cells.

Lung cancers can be divided into two categories: SCLC and NSCLC, which exhibit different morphology, are treated differently and have different prognoses. SCLC is often aggressive with rapid growth and presents with metastasis in distant organs at the time of diagnosis. SCLC has a highly invasive potential. In the present study, we demonstrated a robust difference in expression of CKs between NSCLC and SCLC cell lines. Circulating species related to CK8, 18, and 19 (CK8, 18 and 19 for TPA, CK18 for TPS, and CK19 for Cyfra21-1) have been used as tumor markers in various types of malignancies including NSCLC (2). Consistent with this clinical observation, the present study demonstrated that expression levels of CK8, 18 and 19 were higher in NSCLCs than SCLCs. Since the expression level of CKs was negatively related to invasive potential in the present study, CKs may be used as a factor for determining the phenotypic difference between SCLCs and NSCLCs. In addition, invasive NSCLC cells established by MICS were smaller in size compared with the parental cells (Fig. 2), which more closely resembled the morphology of SCLCs. It is reasonable that invasive NSCLC cells express CKs to a lesser extent since CKs exist throughout the cytoplasm.

We expanded upon our previous observations suggesting the presence of threshold expression levels of dominant CKs in individual cell lines to maintain sufficient CK networks and the importance of redundant expression of multiple CKs for compensation of type I and type II CK pools (4). For example, CK19 is a minor CK found in A549 cells, and suppression of CK19 did not affect the invasive phenotype or the total amount of CKs. In contrast, exogenous CK19 significantly affected the invasiveness of the HI1017 cell line, which does not express endogenous CK19. Suppression of CK18 increased the invasiveness of A549 cells. Since CK18 is dominant in A549, the total amount of CKs was also significantly reduced when CK18 was suppressed. This compensatory mechanism of type I and II CKs is supposedly the reason for the alteration in the invasive ability of A549 cells. Thus, it may be important to regulate the expression of the dominant $\mathrm{CK}$ in each cell line to affect invasive ability.

NSCLC cells can acquire an invasive phenotype during EMT (14). Epithelial markers such as CKs have been reported to decrease in EMT-induced cells (13). Our data are also consistent with the observation that invasive lung cancer cells express CKs at lower levels.

Consistent with our data, it has been reported that oral squamous cell carcinoma cell lines, which do not express CK19, are significantly more invasive in vitro than those which retain expression of this gene (5). In addition, down-regulated expression of CK18 was found to promote the progression of breast cancer (6). Moreover, coordinated co-expression of CK8 and CK18 in a human breast adenocarcinoma cell line was found to dramatically reduce invasiveness and metastatic potential (7).
Another important intermediate filament which may affect invasive ability is vimentin. It is well known that expression of vimentin is generally higher in EMT-induced invasive cells (12). Invasive lung cancer cell lines strongly express CKs and vimentin (11). In contrast, forced CK18 expression was associated with a complete loss of vimentin expression in a parental breast cancer cell line, and resulted in reduced malignant potential (7). Suppression of vimentin by siRNA did not alter the expression of CKs in the HI1017 cells in the present study (data not shown). Mutual correlation of CKs and vimentin on lung cancer invasion should be clarified in future studies

As mentioned above, several CK-related proteins, such as Cyfra21-1, have been used as tumor markers in patients with NSCLC. Additionally, increasing values of serum CK8 have been significantly associated with tumor progression and decreased survival in patients with NSCLC (15). These data contradict our current observation of CKs as negative regulators of cell invasion. However, no data has been reported on the direct correlation regarding the total quantity of tumor cells (i.e. cell number, weight or cubic volume) and serum levels of CK-related species. When a tumor grows, serum concentrations of CKs may also become higher, even when the expression of CKs in each cell does not change. Therefore, we speculate that a higher concentration of serum CKs in advanced cancers may simply reflect an increased tumor burden.

In summary, expression of CKs is negatively associated with the invasiveness of NSCLC cell lines. Expression levels of dominant CKs, but not minor CKs, may affect invasive ability. Regulation of CKs may be useful as a treatment strategy for NSCLC.

\section{Acknowledgements}

The authors thank Ms. Takimi Tamaki for her support of this study.

\section{References}

1. Coulombe PA and Omary MB: 'Hard' and 'soft' principles defining the structure, function and regulation of keratin intermediate filaments. Curr Opin Cell Biol 14: 110-122, 2002.

2. Ishii T, Bandoh S, Fujita J, Ohtsuki Y, Tojo Y, Kanaji N, Fukunaga Y, Ueda Y, Ishida $\mathrm{T}$ and Kubo A: Full-length cytokeratin 8 is released and circulates in patients with non-small cell lung cancer. Tumour Biol 29: 57-62, 2008.

3. Ku NO, Zhou X, Toivola DM and Omary MB: The cytoskeleton of digestive epithelia in health and disease. Am J Physiol 277: G1108-G1137, 1999.

4. Kanaji N, Bandoh S, Fujita J, Ishii T, Ishida T and Kubo A: Compensation of type I and type II cytokeratin pools in lung cancer. Lung Cancer 55: 295-302, 2007.

5. Crowe DL, Milo GE and Shuler CF: Keratin 19 downregulation by oral squamous cell carcinoma lines increases invasive potential. J Dent Res 78: 1256-1263, 1999.

6. Woelfle U, Sauter G, Santjer S, Brakenhoff R and Pantel K: Down-regulated expression of cytokeratin 18 promotes progression of human breast cancer. Clin Cancer Res 10: 2670-2674, 2004.

7. Bühler $\mathrm{H}$ and Schaller G: Transfection of keratin 18 gene in human breast cancer cells causes induction of adhesion proteins and dramatic regression of malignancy in vitro and in vivo. Mol Cancer Res 3: 365-371, 2005.

8. Yamashiro Y, Takei K, Umikawa M, Asato T, Oshiro M, Uechi Y, Ishikawa T, Taira K, Uezato H and Kariya K: Ectopic coexpression of keratin 8 and 18 promotes invasion of transformed keratinocytes and is induced in patients with cutaneous squamous cell carcinoma. Biochem Biophys Res Commun 399: 365-372, 2010 . 
9. Chu YW, Runyan RB, Oshima RG and Hendrix MJ: Expression of complete keratin filaments in mouse L cells augments cell migration and invasion. Proc Natl Acad Sci USA 90: 4261-4265, 1993.

10. Chu YW, Seftor EA, Romer LH and Hendrix MJ: Experimental coexpression of vimentin and keratin intermediate filaments in human melanoma cells augments motility. Am J Pathol 148: 63-69, 1996.

11. Chu YW, Yang PC, Yang SC, Shyu YC, Hendrix MJ, Wu R and $\mathrm{Wu} \mathrm{CW}$ : Selection of invasive and metastatic subpopulations from a human lung adenocarcinoma cell line. Am J Respir Cell Mol Biol 17: 353-360, 1997.

12. Takeyama Y, Sato M, Horio M, Hase T, Yoshida K, Yokoyama T, Nakashima H, Hashimoto N, Sekido Y, Gazdar AF, Minna JD, Kondo $M$ and Hasegawa Y: Knockdown of ZEB1, a master epithelial-to-mesenchymal transition (EMT) gene, suppresses anchorage-independent cell growth of lung cancer cells. Cancer Lett 296: 216-224, 2010.
13. Kasai H, Allen JT, Mason RM, Kamimura T and Zhang Z: TGF-betal induces human alveolar epithelial to mesenchymal cell transition (EMT). Respir Res 6: 56, 2005.

14. Keshamouni VG, Michailidis G, Grasso CS, Anthwal S, Strahler JR, Walker A, Arenberg DA, Reddy RC, Akulapalli S, Thannickal VJ, Standiford TJ, Andrews PC and Omenn GS: Differential protein expression profiling by iTRAQ-2DLC-MS/ MS of lung cancer cells undergoing epithelial-mesenchymal transition reveals a migratory/invasive phenotype. J Proteome Res 5: 1143-1154, 2006.

15. Fukunaga Y, Bandoh S, Fujita J, Yang Y, Ueda Y, Hojo S, Dohmoto K, Tojo Y, Takahara $\mathrm{J}$ and Ishida $\mathrm{T}$ : Expression of cytokeratin 8 in lung cancer cell lines and measurement of serum cytokeratin 8 in lung cancer patients. Lung Cancer 38: 31-38, 2002. 